\title{
LIVRE BLANC \\ DE LA RECHERCHE EN MÉCANIQUE
}

\section{Enjeux industriels et sociétaux Recherche, innovation, formation}

\section{Association Française de Mécanique}

Février 2015
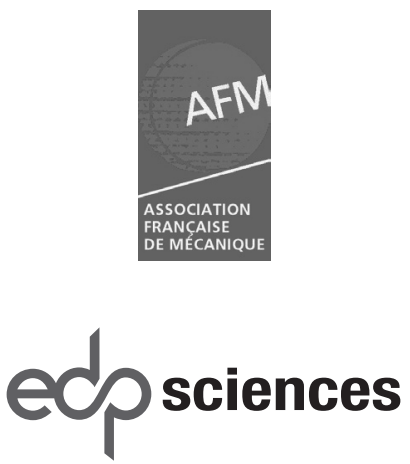

17, avenue du Hoggar

Parc d activités de Courtaboeuf, BP 112

91944 Les Ulis Cedex A, France 
L a filière mécanique est une pierre angulaire de l'industrie. Elle constitue à la fois un secteur industriel à part entière (l'industrie mécanique représentée par les métiers de l'équipement, de la transformation et de la précision regroupés au sein de la FIM, Fédération des Industries Mécaniques), mais aussi un ensemble de technologies sur lesquelles se fondent de nombreux secteurs tels que ceux qui construisent des matériels pour les transports, l'énergie, la santé, l'environnement.

Ce Livre Blanc de la Recherche en Mécanique est élaboré par le Haut Comité Mécanique (HCM) dans le cadre de l'Association Française de Mécanique (AFM). Ses objectifs sont : I'identification des besoins industriels et des technologies innovantes, la proposition des orientations de recherche et technologie pour faire initier des projets R\&D, et l'apport de l'information pour des industriels, des décideurs mais aussi des scientifiques qui peuvent ignorer les ressources que recèle leur milieu.

Quatre grands chapitres composent ce Livre Blanc. Après la présentation des éléments du panorama, les grands enjeux dans les secteurs industriels sont détaillés, les défis scientifiques sont traités par thématiques de recherche et les approches qui permettent de concevoir et produire autrement sont abordées. On trouve, en annexe du livre, une liste des organismes et des laboratoires de recherche dans le domaine de la Mécanique.

Ce livre est un ouvrage collectif de 114 auteurs. Le comité de rédaction, Michel Lebouché, président du HCM, Mansour Afzali, Pierre Devalan et Claude Hauviller, a rassemblé et structuré les contributions d'individuels, des groupes de travail de l'AFM et des sociétés savantes. Il a été publié avec l'aide du Centre Technique des Industries Mécaniques et de la Fédération des Industries Mécaniques.

Imprimé en France

ISBN : 978-2-7598-1683-5

DOI : 10.1051/978-2-7598-1683-5

Cet ouvrage est publié en Open Access sous licence creative commons CC-BY-NC-ND (https:// creativecommons.org/licenses/by-nc-nd/4.0/fr/) permettant l'utilisation non commerciale, la distribution, la reproduction du texte, sur n'importe quel support, à condition de citer la source. 